\begin{tabular}{|c|l|}
\hline Title & UItrahigh quality factor in a metal-embedded semiconductor microdisk cavity \\
\hline Author(s) & Kurosawa, Hiroyuki; Kumano, Hidekazu; Suemune, Ikuo \\
\hline Citation & $\begin{array}{l}\text { Optics Letters, } 40 \text { (24), 5766-5769 } \\
\text { https:/doi.org/40.1364/0L.40.005766 }\end{array}$ \\
\hline Issue Date & 2015-12-15 \\
\hline Doc URL & http://hdl.handle.net/2115/63822 \\
\hline Rights & ○ 2015 Optical Society of A merica \\
\hline Type & article (author version) \\
\hline File Information & Kumano_OpLet2015.pdf \\
\hline
\end{tabular}

Instructions for use 


\title{
Ultra-high Quality Factor in a Metal-embedded Semiconductor Microdisk Cavity
}

\author{
HIROYUKI Kurosawa ${ }^{1 *}$, HideKAZU Kumano ${ }^{1}$, ANd IKUO SUEmUne ${ }^{1}$ \\ ${ }^{1}$ Research Institute for Electronic Science, Hokkaido University, Sapporo, 001-0021, Japan \\ *KUROSAWA.Hiroyuki@nims.go.jp
}

Compiled November 13, 2015

\begin{abstract}
We numerically and theoretically investigate electrodynamics of a metal-embedded semiconductor microdisk cavity. The electrodynamics of a cavity mode is discussed from the viewpoint of quantum mechanics, which clarifies the condition for high $Q$ factor. Using numerical calculation, we optimize the cavity structure and show that the $Q$ factor can be increased up to $1,700,000$. Our study suggests that the metal-embedded cavity is a promising candidate for cavity quantum electrodynamics devices. () 2015 Optical Society of America
\end{abstract}

OCIS codes: (000.1600) Classical and quantum physics; (140.3945) Microcavities; (140.4780) Optical resonators; (260.2110) Electromagnetic optics; (350.3950) Micro-optics

http://dx.doi.org/10.1364/ao.XX.XXXXXX

Quality factor ( $Q$ factor) of cavity has been the focus of interest in optics, photonics and optoelectronics since the Purcell's discovery that spontaneous emission can be controlled by manipulating the photonic density of states with a cavity [1]. Various kinds of cavities have been proposed and demonstrated in order to achieve higher $\mathrm{Q}$ factor and smaller mode volume. For example, pillar microcavities based on distributed Bragg reflectors (DBRs) [2], microdisk ( $\mu$-disk) [3], sphere [4], toroidal [5] resonators, and photonic crystal cavities [6] are now available as a cavity with high $Q$ factor. Even though these cavities have high $\mathrm{Q}$ factors and small mode volumes, whole cavity structures generally occupy a relatively large amount of space compared with operating wavelengths. This relatively large cavity volume is basically due to the property of dielectric open cavities. On the other hand, metallic nanocavites are able to achieve small cavity volumes due to the metallic nature of strong confinement of electromagnetic (EM) fields.

Metallic nanocavities have been extensively studied for realizing nanolasers with miniaturized cavity volumes [7-11]. However, the cavity $\mathrm{Q}$ factors measured up to now remains low below $\sim 500$ due to significant optical absorption of the constituent metals [7-11]. The metallic nanocavities have also been numerically characterized and the higher $Q$ factors of $\sim 1700$ [12] and $\sim 3400$ [13] have been reported. Recently, higher $Q$ factor about 5000 at room temperature [14] and 9000 at $20 \mathrm{~K}$ [15] have been demonstrated in a metal-embedded cavity. Al- though high $\mathrm{Q}$ factor in a metallic cavity has been experimentally demonstrated, the generation mechanism of the high $Q$ factor is not fully clarified so far.

In this Letter, we present a theoretical and numerical study of high $\mathrm{Q}$ factor in a metal-embedded semiconductor $\mu$-disk cavity. The electrodynamics of high $\mathrm{Q}$ mode is analyzed from the viewpoint of quantum mechanical particle subject to an effective potential. Using this analysis, we reveal crucial parameters to achieve higher $Q$ factor. Following the theoretical study, we propose an optimized metal-embedded cavity and numerically explore high-Q cavity mode. We demonstrate a cavity mode with an ultra-high $Q$ factor of 1,700,000.

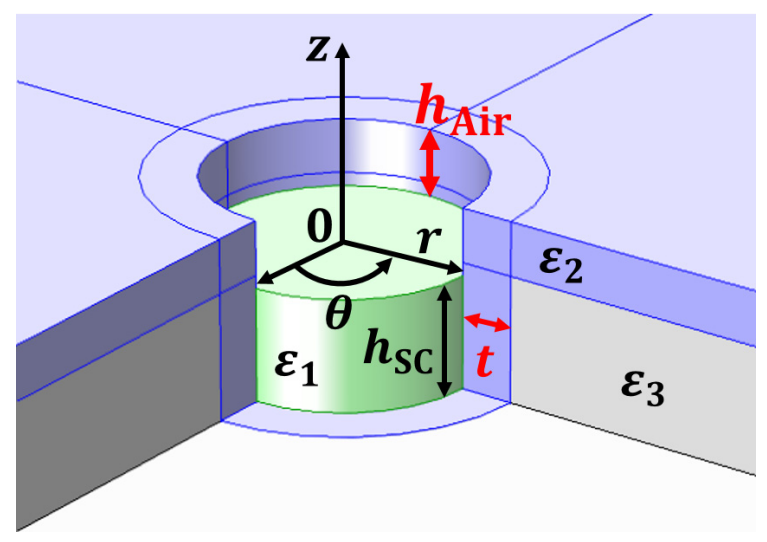

Fig. 1. (Color online) Schematic of the metal embedded semiconductor $\mu$-disk cavity with transparent dielectric spacer. The radius and height of the cavity is denoted by $r$ and $h_{\mathrm{SC}}$, respectively. The subscript SC in $h_{\mathrm{SC}}$ means the shorthand of semiconductor.

Let us consider a metal-embedded cavity shown in Fig. 1. The cavity consists of a homogeneous semiconductor $\mu$-disk with permittivity $\varepsilon_{1}$ and radius $r$. We assume that the $\mu$-disk is embedded in a metallic medium with permittivity $\varepsilon_{3}$. The metallic material is assumed to have small optical loss in the visible and near infrared regions. We insert a transparent dielectric spacer between the $\mu$-disk and the metallic medium. The thickness of the dielectric spacer is $t$. As a numerical example, we assume that the semiconductor $\mu$-disk, the transparent dielectric spacer and the metallic material consist of GaAs, silica $\left(\mathrm{SiO}_{2}\right)$ 
and silver $(\mathrm{Ag})$, respectively. The refractive index of GaAs $\left(n_{\mathrm{GaAs}}\right)$ and $\mathrm{SiO}_{2}\left(n_{\mathrm{SiO}_{2}}\right)$ are set to 3.5 and 1.46 , respectively. The permittivity of $\mathrm{Ag}$ was taken from the literature [16]. We also assume the wavelength of light is $1000 \mathrm{~nm}$. These parameters are similar to those in [14]. The computational domain is terminated by impedance boundary condition. Throughout this Letter, we performed numerical calculation based on a finite element method (FEM) using COMSOL Multiphysics. Since the resonance condition with respect to cavity structure is related to the radius $(r)$ and height $\left(h_{\mathrm{SC}}\right)$ of the GaAs $\mu$-disk, we fix these parameters and set $r$ and $h_{\mathrm{SC}}$ to $500 \mathrm{~nm}$ and $300 \mathrm{~nm}$, respectively. Hence, we investigate the role of the height of the air region and thickness of silica in $\mathrm{Q}$ factor. We first investigate the role of the height of the air region in $Q$ factor. Figure 2(a) shows $Q$ factor of the cavity as a function of the height of the air region. In this numerical calculation, we set the thickness of the silica spacer to $260 \mathrm{~nm}$. The $\mathrm{Q}$ factor rapidly increases with the height of the air region. The $Q$ factor curve also has a saturation feature when the core of the cavity is deeply embedded in a metallic material. In this Q-factor curve, the cavity mode is whispering gallery mode (WGM) with mode number 7 (see the inset in Fig. 2(a)). These features are elucidated as follows. The height of the air region plays the role of coupling parameter of cavity mode to free space. In other words, the height of the air region can control the radiation loss from the top surface of the cavity. With increasing the height, the radiation loss to free space decreases. Therefore, the light confinement increases with the height, which results in the enhancement of $Q$ factor. When the height of the air region exceeds a critical value, the radiation loss from the cavity is minimized, which is the reason of the saturation feature of the $Q$ factor in Fig. 2(a).

We next investigate the role of the thickness of the silica spacer in $Q$ factor. Figure 2(b) shows $Q$ factor of the cavity as a function of the thickness of the silica spacer. In this numerical calculation, we set the height of the air region to 700 $\mathrm{nm}$. There are two remarkable features in the Q-factor curve. With increasing the thickness of the silica spacer, the $Q$ factor increases. When the thickness exceeds a critical value $(260 \mathrm{~nm}$ in Fig. 2(b)), the $Q$ factor decreases. Namely, there exits an optimum value of silica thickness. In order to elucidate this feature, let us start with the vector Helmholtz equation in cylindrical coordinates: $\left[\nabla^{2}+(\omega / c)^{2} \varepsilon_{i}\right] \vec{E}(\vec{r})=0$, which is modified to the scalar Helmholtz equation

$$
\left(\frac{1}{r} \frac{\partial}{\partial r}\left(r \frac{\partial}{\partial r}\right)+\frac{1}{r^{2}} \frac{\partial^{2}}{\partial \theta^{2}}+\frac{\partial^{2}}{\partial z^{2}}+\frac{\omega^{2}}{c^{2}} \varepsilon_{i}\right) f(\vec{r})=0,
$$

where $f(\vec{r})=\vec{r} \cdot \vec{E}, \varepsilon_{i}$ is the permittivity of material in $i$ th region, and we assumed transverse wave : $\nabla \cdot \vec{E}=0$ [17]. Here, we assume the $\mu$-disk is infinite in the $z$ direction. Namely, we consider an infinite cylinder in the $z$ direction. We also introduce the azimuth angle dependence of the wave function as $e^{-i l \theta}$, where $l$ is an integer. Moreover, we convert the radial wave function $R(r)$ to the form $u(r) / \sqrt{r}$. Then, we obtain the wave equation for the modified radial function $u(r)$ as

$$
\frac{d^{2} u(r)}{d r^{2}}-\frac{l^{2}-1 / 4}{r^{2}} u(r)+\left(\frac{\omega}{c}\right)^{2} \varepsilon_{i} u(r)=0 .
$$

Here, formally introducing the virtual mass of photon as $m^{*}$ and multiplying $-\hbar^{2} / 2 m^{*}$ on the both side of Eq. (2), we can modify the wave equation to the form:

$$
-\frac{\hbar^{2}}{2 m^{*}} \frac{d^{2} u(r)}{d r^{2}}+V_{\mathrm{eff}}^{(i)} u(r)=E u(r),
$$

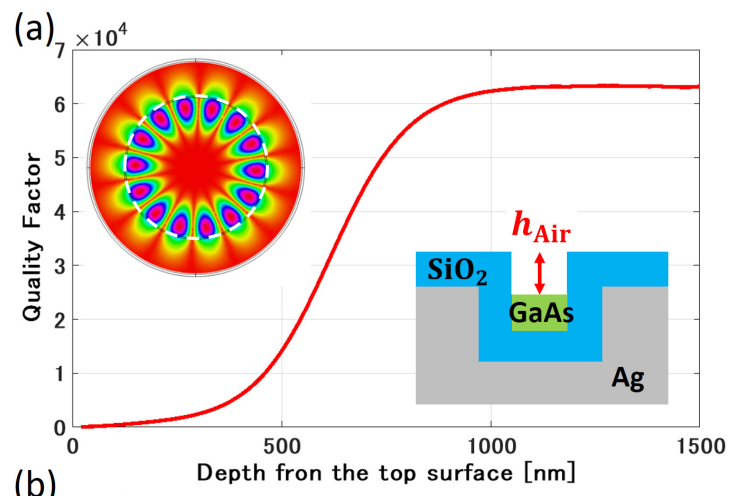

(b)

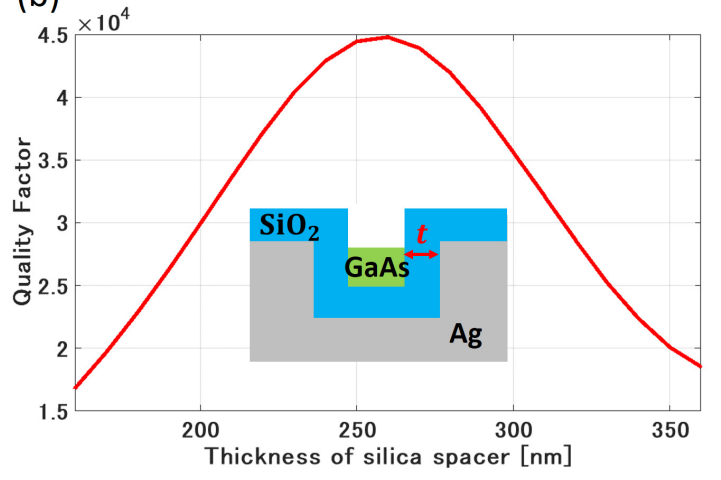

Fig. 2. (Color online) Quality factor as as function of the height of the air region (a) and the thickness of the silica spacer (b). The upper left inset in Fig. 2(a) shows the electric field intensity distribution of the cavity mode. The other insets show the schematic of the vertical cross-section of the cavity.

where

$$
\begin{aligned}
V_{\mathrm{eff}}^{(i)} & =\frac{\hbar^{2}}{2 m^{*}}\left[\left(\frac{\omega}{c}\right)^{2}\left(1-\varepsilon_{i}\right)+\frac{l^{2}-1 / 4}{r^{2}}\right], \\
E & =\frac{\hbar^{2}}{2 m^{*}}\left(\frac{\omega}{c}\right)^{2} .
\end{aligned}
$$

Equation (3) has the formally-same form as the Schrödinger equation in 1 dimension. Therefore, electrodynamics in the cylinder which is infinite in the $z$ direction is equivalent to the dynamics of a quantum mechanical particle subject to the effective potential. Henceforth, we move on the particle picture for easier understanding of the cavity-mode dynamics.

As an example of the effective potential, we consider the potential corresponding to the situation that a GaAs cylinder with radius $500 \mathrm{~nm}$ coated by $\mathrm{SiO}_{2}$ is embedded in $\mathrm{Ag}$. The material parameters of $\mathrm{GaAs}, \mathrm{SiO}_{2}$ and $\mathrm{Ag}$ are the same as those of the previous FEM calculation. We also set the wavelength of light and the azimuth number are $1000 \mathrm{~nm}$ and 7, respectively. Since $\hbar^{2} /\left(2 m^{*}\right)$ is a common factor of the effective potential and the energy level of light, it is convenient to consider the ratio between them. Then, we consider two cases: one is the case with thin $\mathrm{SiO}_{2}$ spacer, the other is that of thick $\mathrm{SiO}_{2}$ spacer. Under those parameters, we show the schematic of effective potential and the kinetic energy of the particle as a function of distance from the center of the cylinder in Fig. 3. In Fig. 3(a), we set the thickness of $\mathrm{SiO}_{2}$ to $100 \mathrm{~nm}$, which is much thinner than the critical thickness obtained in Fig. 2(b). Around the GaAs/Silica interface, the effective potential curve in Fig. 3(a) has a valley of the potential under the energy level of light bounded 
by higher potential barriers. Namely, a bound state is formed around the interface. The bound state of light corresponds to a confined EM mode. A wave function of the bound state decays exponentially from the GaAs region. The penetration depth of the wavefunction $\left(\sim \lambda / 2 n_{\mathrm{SiO} 2}\right)$ is much longer than the silica spacer. Therefore, the wavefunction has high existence probability near the Silica/Ag interface. Hence, the particle near the Silica/Ag interface is strongly absorbed in the Ag region due to the ohmic loss of the metal, which results in low $\mathrm{Q}$ factor. Therefore, we conclude that thicker dielectric spacer is preferable for the reduction of metal absorption loss in $\mathrm{Q}$ factor.

Based on the above discussion, we set the thickness of the spacer to much thicker value. In Fig. 3(b), we set the thickness of $\mathrm{SiO}_{2}$ spacer to $760 \mathrm{~nm}$, which is much thicker than the critical thickness. There are two valleys under the energy level of light bounded by higher potential barriers. One is around the GaAs/Silica interface and the other is Silica/Ag interface. Namely, two bound states are formed in this situation. From the viewpoint of quantum mechanics, we find the latter bound state is formed due to tunneling effect. The tunneling of light corresponds to the radiation from the cavity. The bound state near the GaAs/Silica interface decays exponentially from the interface and has low existence probability in the Ag region. Hence, the bound state near the GaAs/Silica does not reduce $Q$ factor. However, the other bound state near the Silica / Ag interface has high existence probability near the interface. Hence, the $Q$ factor of this situation is low as well as the case of thin spacer. Using the effective potential, we find that the silica spacer is preferable to be thick but should be thin so that the tunneling of the particle cannot occur. Therefore, the optimum thickness of the silica is given by the point at the intersection of effective potential with the energy level of light. We show the corresponding configuration of the effective potential and the energy level of light in Fig. 3(c), where the thickness of the silica spacer is 298 $\mathrm{nm}$. This thickness is close to the critical thickness in Fig. 2. In this configuration, there is a bound state near the GaAs/Silica interface. The bound state is located away from the Ag region. Moreover, the tunneling effect of light is prevented due to the high potential barrier of Ag. All these features contribute to higher Q factor.

Let us confirm the validity of our discussion by numerical calculation. According to the previous discussion, the GaAs core should be deeply embedded in $\mathrm{Ag}$ so that the radiation loss from the top surface of the cavity is minimized. The thickness of the spacer between GaAs and Ag is preferable to be thick as long as the tunneling effect of light does not occur. Moreover, the effective potential analysis suggests that the permittivity of the transparent dielectric spacer should be small. The smaller permittivity in the dielectric spacer region makes the potential barrier in the region higher, which enhances the confinement of light in the GaAs core. Based on the above analysis, we optimized the cavity structure to increase the $Q$ factor. We show the result in Fig. 4. Figures 4(a) and (b) show the schematic of the optimized metal-embedded cavity structure. The GaAs $\mu$-disk is on silica layer, which is basically the same structure as that of Fig. 1. The main difference is that the lateral $\mu$-disk surface is surrounded by air. The structure parameters are shown in Fig. 4(b). $h_{\mathrm{Air}}, h_{\mathrm{SC}}$ and $h_{\mathrm{SiO}_{2}}$ shown in Fig. $4(\mathrm{~b})$ are $600 \mathrm{~nm}, 600 \mathrm{~nm}$, and $450 \mathrm{~nm}$, respectively. The thickness of the air spacer, $t_{\mathrm{Air}}$, is $430 \mathrm{~nm}$. This value is much thicker than the optimum value 260 nm given in Fig. 2(b). The optimum thickness of the spacer increased without allowing the tunneling effect of light, which is due to the higher potential barrier in the air spacer region. Fig-
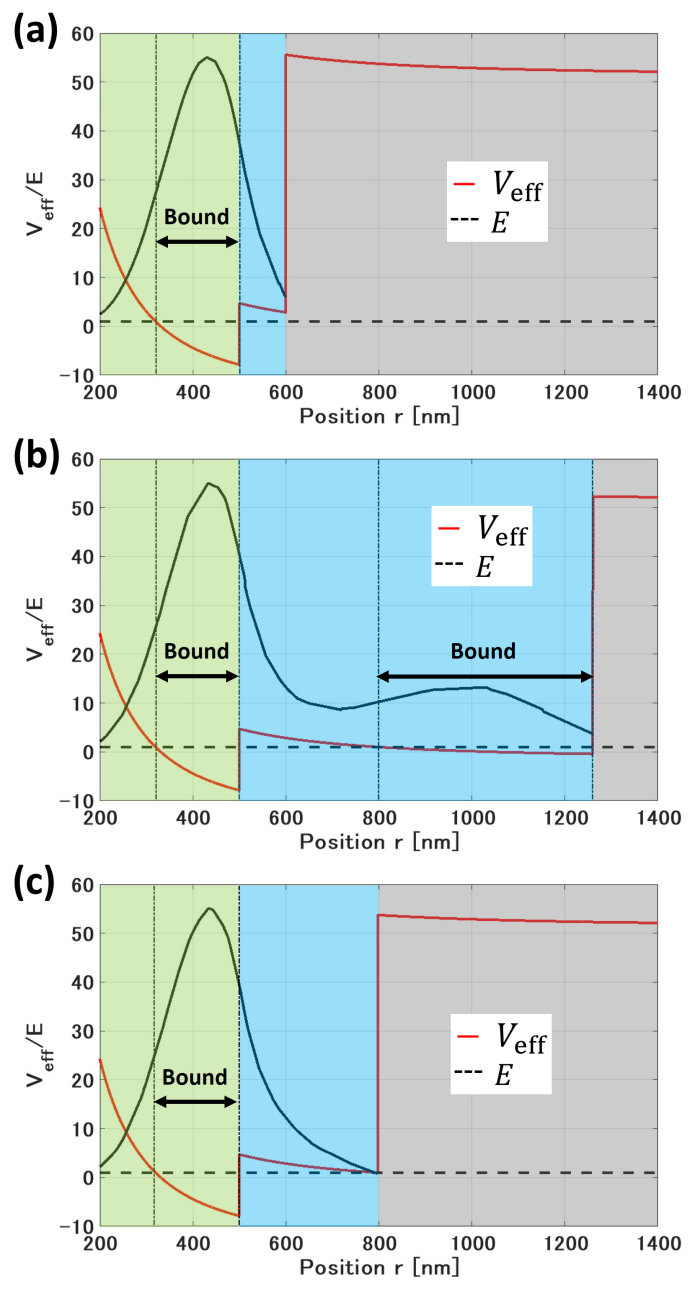

Fig. 3. (Color online) Effective potential as a function of radius of the $\mu$-disk cavity for the case of thin (a), thick (b) and optimum (c) spacers. The potential curve and the energy level of light are drawn by the red and broken black lines, respectively. The green-, blue- and gray-shaded area correspond to the GaAs, silica and Ag regions, respectively. The spatial profiles of the cavity mode are drawn by black lines.

ures 4(c) and (d) show the electric field intensity distribution of the optimized cavity mode. The cavity mode is TE-polarized and its mode number is 7 . The field distribution indicates that the photon in the cavity is strongly confined in the GaAs $\mu$-disk. Moreover, the intensity of the cavity mode inside the $\mu$-disk becomes maximum away from the lateral surface of the $\mu$-disk. This field distribution is preferable for reduction of the surface scattering loss. The $\mathrm{Q}$ factor of the cavity mode is calculated to be about $1,700,000$. In this ultra-high $Q$ factor, we estimated $Q$ factors attributed from the radiation at the top surface of the cavity and effective metal absorption loss at $1.0 \times 10^{8}$ and $1.7 \times 10^{6}$, respectively. This ultra-high $\mathrm{Q}$ factor indicates that the metallic loss of the cavity mode can be strongly reduced by optimizing the material parameters of the dielectric spacer region. As well as $\mathrm{Q}$ factor, we focus on mode volume of the cavity mode. Figure 5 shows the $\mathrm{Q}$ factor and mode volume of the metal-embedded cavity as a function of mode number of WGM. In this numerical calculation, we only changed the radius of the optimized cavity. We find that the metal-embedded 


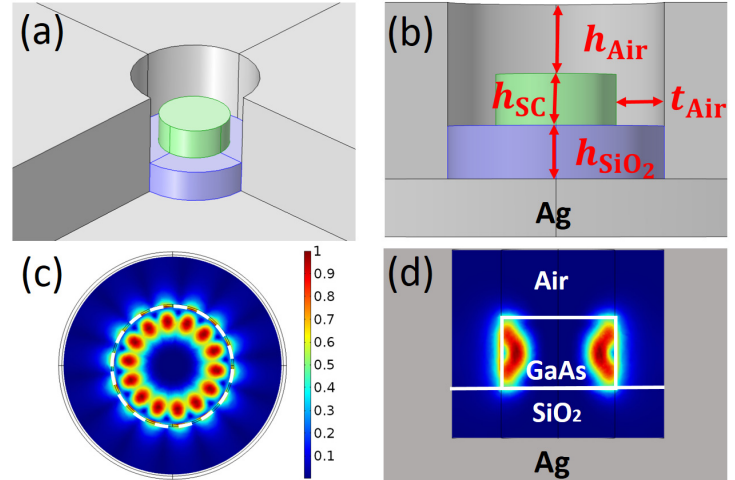

Fig. 4. (Color online) Bird's eye view (a) and cross-section view (b) of the optimized cavity structure. The green and blue $\mu$-disks consist of GaAs and silica, respectively. Top view (c) and lateral cross-sectional view (d) of the electric field distribution of the calculated ultra-high-Q cavity mode.

cavity has several ultra-high $\mathrm{Q}$ factors and the mode volumes are comparable with $\left(\lambda / n_{\mathrm{GaAs}}\right)^{3}$. This result indicates that the metal-embedded cavity can achieve both of ultra-high $Q$ and small mode volume.

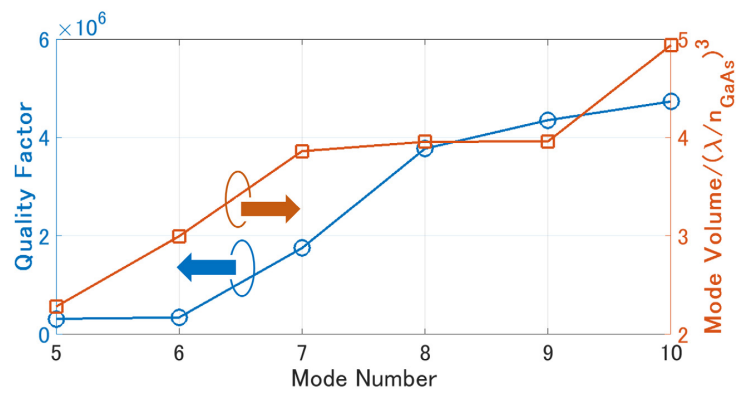

Fig. 5. (Color online) Quality factor (blue) and mode volume (red) of the metal-embedded cavity as a function of mode number of WGM.

The obtained ultra-high-Q cavity mode is TE-polarized. It is also worth considering the polarization dependence of the $\mathrm{Q}$ factor. Since the particle picture based on the Shrödingerlike equation (3) cannot include the polarization of cavity mode, we consider the polarization dependence of the $Q$ factor from the viewpoint of the Maxwell boundary condition (MBC). The $\mathrm{MBC}$ requires the continuity of the vertical component of electric displacement across a boundary: $\varepsilon^{M} E_{\perp}^{M}=\varepsilon^{D} E_{\perp}^{D}$, where the superscripts $M$ and $D$ indicate a quantity in the metallic and dielectric regions, respectively. The symbol $\perp$ indicates the vertical component of the labeled quantity. Namely, the electric field in the metallic region is strongly reduced due to high permittivity of metallic material. This reduction of electric field in the metallic material is preferable for high $\mathrm{Q}$ factor. Therefore, the cavity mode with TE polarization has high $Q$ factor in a metal-embedded cavity.

In conclusion, we numerically and theoretically investigated electrodynamics in a metal-embedded semiconductor $\mu$-disk cavity. In particular, we analyzed the radiation and metallic absorption loss from the viewpoint of quantum mechanical particle subject to an effective potential. We showed that there exists a critical thickness of the dielectric spacer to reduce the metallic absorption loss of cavity mode, which is the consequence of the tunneling effect of light and a bound state near the metal region. Based on these findings, we proposed the optimized cavity structure that increases the effective potential barrier. We demonstrated that the $\mathrm{Q}$ factor of the cavity mode can be increased up to 1,700,000 in the optimized metallic cavity. Our numerical study suggested that the metal-embedded cavity has a potential to show ultra-high $\mathrm{Q}$ factor. Moreover, we would like to note that the metal-embedded cavity has high extraction efficiency of single photons emitted from a semiconductor quantum dot placed inside the similar metallic nanostructure [18, 19]. We can control both of the $\mathrm{Q}$ factor of the cavity mode and the photon extraction through the radiation to surface by adjusting the height of the air region. These features indicate that the metal-embedded cavity is one of the promising candidates for cavity quantum electrodynamics (QED) devices.

\section{FUNDING INFORMATION}

Funding. Grant-in-Aid for Young Scientists (Start-up) Grant Number 25889001 from JSPS KAKENHI.

\section{REFERENCES}

1. E. M. Purcell, H. C. Torrey, and R. V. Pound, Phys. Rev. 69, 37 (1946).

2. J. M. Gérard, B. Sermage, B. Gayral, B. Legrand, E. Costard, and V. Thierry-Mieg, Phys. Rev. Lett. 81, 1110 (1998).

3. M. Soltani, S. Yegnanarayanan, and A. Adibi, Opt. Express 15, 4694 (2007).

4. D. W. Vernooy, V. S. Ilchenko, H. Mabuchi, E. W. Streed, and H. J. Kimble, Opt. Lett. 23, 247 (1998).

5. D. K. Armani, T. J. Kippenberg, S. M. Spillane, and K. J. Vahala, Nature 421, 925 (2003).

6. O. Painter, J. Vučkovič, and A. Scherer, J. Opt. Soc. Am. B 16, 275 (1999).

7. M. T. Hill, Y.-S. Oei, B. Smalbrugge, Y. Zhu, T. de Vries, P. J. van Veldhoven, F. W. M. van Otten, T. J. Eijkemans, J. P. Turkiewicz, H. de Waardt, E. J. Geluk, S.-H. Kwon, Y.-H. Lee, R. Notzel, and M. K. Smit, Nat Photon 1, 589 (2007).

8. M. T. Hill, M. Marell, E. S. P. Leong, B. Smalbrugge, Y. Zhu, M. Sun, P. J. van Veldhoven, E. J. Geluk, F. Karouta, Y.-S. Oei, R. Nötzel, C.-Z. Ning, and M. K. Smit, Opt. Express 17, 11107 (2009).

9. C.-Y. Lu, S.-W. Chang, S. L. Chuang, T. D. Germann, and D. Bimberg, Applied Physics Letters 96, 251101 (2010).

10. M. Khajavikhan, A. Simic, M. Katz, J. H. Lee, B. Slutsky, A. Mizrahi, V. Lomakin, and Y. Fainman, Nature 482, 204 (2012).

11. K. Ding, M. T. Hill, Z. C. Liu, L. J. Yin, P. J. van Veldhoven, and C. Z. Ning, Opt. Express 21, 4728 (2013).

12. J. H. Lee, M. Khajavikhan, A. Simic, Q. Gu, O. Bondarenko, B. Slutsky, M. P. Nezhad, and Y. Fainman, Opt. Express 19, 21524 (2011).

13. M. P. Nezhad, A. Simic, O. Bondarenko, B. Slutsky, A. Mizrahi, L. Feng, V. Lomakin, and Y. Fainman, Nat Photon 4, 395 (2010).

14. R. Takemoto, N. Ishihara, H. Kurosawa, N. A. Jahan, T. Asano, X. Liu, H. Nakajima, H. Kumano, and I. Suemune, Applied Physics Letters 103, 191104 (2013).

15. N. Ishihara, H. Kurosawa, R. Takemoto, N. A. Jahan, H. Nakajima, H. Kumano, and I. Suemune, Nanotechnology 26, 085201 (2015).

16. A. D. Rakić, A. B. Djurišić, J. M. Elazar, and M. L. Majewski, Appl. Opt. 37, 5271 (1998).

17. L. Novotny and B. Hecht, Principles of Nano-Optics, 2nd Edition (Cambridge University Press, 2012).

18. H. Kumano, H. Nakajima, H. lijima, S. Odashima, Y. Matsuo, K. ljiro, and I. Suemune, Applied Physics Express 6, 062801 (2013).

19. X. Liu, T. Asano, S. Odashima, H. Nakajima, H. Kumano, and I. Suemune, Applied Physics Letters 102, 131114 (2013). 